\section{Are all rTMS machines equal? New research suggests there may be clinically significant differences}

\section{Will Novey \\ UC Irvine School of Medicine, Irvine, CA, USA}

Technological advancements in psychiatry seldom occur. Unlike many surgical and medical subspecialties, psychiatry relies heavily on the role of pharmacology and talk therapy to guide patient care. In the 1930 s, the advent of Electroconvulsive therapy (ECT) revolutionized psychiatry by physically changing brain activity via interfacing with a machine. However, ECT's unrealistic characterizations from TV and Cinema perpetuated its stigmatization. Even medical students are susceptible to the influence of movie clips of ECT and are less inclined to recommend it as treatment. ${ }^{1}$

In the 1980s, neuroscientist Anthony Barker began experimenting with the effects of magnetic fields on brain activity and in turn developed the first transcranial magnetic stimulation (TMS) device, a tool that provides many of the benefits of ECT with almost none of the side effects. ${ }^{2}$ At first indicated only for research and diagnostics, by 1995 clinical evidence for the use of TMS in treating major depression appeared. ${ }^{3}$ In 2007, O'Reardon et al. conducted a double-blind, multisite study of 301 medication-free patients with refractory depression which revealed that those treated with TMS of the left dorsolateral prefrontal cortex (DLPFC) had statistically significant improvement in depressive symptoms when compared to those treated with sham TMS devices. ${ }^{4}$ This study allowed the researchsponsor, Neuronetics Inc, to gain FDA approval for the NeuroStar TMS device. Additional studies, including a multi-site trial by the NIH, demonstrated that when applied to the DLPFC, repetitive TMS (rTMS) at high frequencies produces excitation through long-term potentiation and provides clinically significant outcomes in treating patients with major depressive disorder (MDD). ${ }^{5}$

Since the release of the NeuroStar, additional TMS devices have gained FDA approval for depression treatment including: Apollo, Bransway Deep, CloudTMS, Magstim, MagVenture, Neurostar, and Nexstim. Unfortunately, little research has been done to compare these devices and their clinical effectiveness. A 2016 retrospective study by Oliveira-Maia et al. compared the antidepressant efficacy of
Magstim vs NeuroStar devices in patients suffering from medication-resistant MDD and found them to be equal in efficacy; however, new research suggests variations in rTMS parameters could be clinically significant. 6

A recently-published paper found there to be a significant difference in the average time to remission for MDD between the NeuroStar and MagVenture machines. ${ }^{7}$ The study was conducted at AwakeningsKC Clinical Neuroscience Institute (CNI), a suburban tertiary psychiatric clinic, using PHQ-9 scores and Cox Proportional Hazard and Log-Rank statistics to compare the remission rates of depression between the two machines. This study, the second of a series of three, ${ }^{8-9}$ found that patients treated using the MagVenture instrument experienced a shorter mean time to remission, higher overall 6-week remission rates, and augmented response rates to CBT. These conclusions raise the question: Are rTMS machines equivalent, and if not, which device should be the standard of care?

The NeuroStar and MagVenture machines vary in features and technical parameters such as cooling mechanisms (MagVenture has one, NeuroStar does not), coil composition (Cool B65 Butterfly Coil $v s$. Iron ferromagnetic), and pulse width (280 S vs $185 \mathrm{~S}$ ). Additionally, the NeuroStar has a higher max acoustic output (93.9 $\mathrm{dB}$ vs $83.4 \mathrm{~dB}$ ) and some patients have reported that the NeuroStar was more painful at the site of application. However, it's unclear how these parameters may directly influence clinical outcome.

There are significant limitations with this study and while it introduced an important question, more evidence is necessary to draw appropriate conclusions about the impact of machine differences on MDD treatment. For instance, patients treated with the MagVenture had significantly higher baseline PHQ-9 scores than those treated with NeuroStar. Therefore, purported differences in instruments on treatment efficacy may actually reflect preference for rTMS use in relation to severity of depression, i.e., patients with more severe depression may respond better to rTMS. The study was also conducted at a suburban facility without any outside recruitment, so a sample of 247 patients ( $97 \%$ Caucasian) may lack external validity. Most importantly, this study was a non-randomized retrospective review of medical records. It would be unwise to make casual inferences from this study without a more thorough investigation.

This study comparing the NeuroStar and MagVenture provides some insight for further research, as the question of clinical
Correspondence: Will Novey, UC Irvine School of Medicine, 1001 Health Sciences Rd, Irvine, CA 92617, USA

Tel.: +1.510.3042907.

E-mail: wnovey@uci.edu

Key words: transcranial magnetic stimulation, TMS machines, clinical differences.

Conflict of interest: the author declares no potential conflict of interest.

Funding: none

Received for publication: 27 March 2019

Accepted for publication: 27 March 2019.

This work is licensed under a Creative Commons Attribution-NonCommercial 4.0 International License (CC BY-NC 4.0).

(C) Copyright W. Novey, 2019

Licensee PAGEPress, Italy

Mental Illness 2019; 11:8125

doi:10.4081/mi.2019.8125

variability between rTMS machines should be further investigated with a large, multicenter, prospective, randomized clinical trial. If there are clinically significant differences in rTMS machines, practitioners should be made aware of this discrepancy as this may influence patient outcomes. The FDA has provided guidelines on how to characterize rTMS devices based on coil positioning, magnetic field characteristics, output waveform, magnetic field spatial distribution, etc., but there remains a paucity of data providing a detailed comparison of each device. 10 Such data is essential for practitioners to make effective clinical decisions.

\section{References}

1. Walter G, McDonald A, Rey JM, Rosen A. Medical student knowledge and attitudes regarding ECT prior to and after viewing ECT scenes from movies. J EcT 2002;18:43-6.

2. Horvath JC, Perez JM, Forrow L, et al. Transcranial magnetic stimulation: a historical evaluation and future prognosis of therapeutically relevant ethical concerns. J Med Ethics 2011;37:13743.

3. Kolbinger HM, Höflich G, Hufnagel A, et al. Transcranial magnetic stimulation (TMS) in the treatment of major depression: a pilot study. Hum Psychopharmacol Clin Exp 1995;10: 305-10.

4. O'Reardon JP, Solvason HB, Janicak PG, et al. Efficacy and safety of tran- 
scranial magnetic stimulation in the acute treatment of major depression: a multisite randomized controlled trial. Biol Psychiatry 2007;62:1208-16.

5. George MS, Lisanby SH, Avery D, et al. Daily left prefrontal transcranial magnetic stimulation therapy for major depressive disorder: a sham-controlled randomized trial. Arch Gen Psychiatry 2010;67:507-16.

6. Garcia-Guarniz AL, Sinanis A, PascualLeone A, Press D. Comparative efficacy of repetitive transcranial magnetic stimulation for treatment of depression using 2 different stimulation devices: A retrospective open-label study. J Clin
Psychiatry 2016;77:e743.

7. Manzardo AM, Ely B, Davila MC. Time to remission analysis for major depressive disorder after repetitive transcranial magnetic stimulation (rTMS). Ment Illn 2019;11:8141.

8. Davila MC, Ely B, Manzardo AM. Repetitive transcranial magnetic stimulation (rTMS) using different TMS instruments for major depressive disorder at a suburban tertiary clinic. Ment Illn 2019;11:7947.

9. Davila MC, Ely B, Manzardo AM. Relationship between body mass and clinical response to repetitive transcranial magnetic stimulation (rTMS) for major depressive disorder. Ment Illn 2019;11:8116

10. Costello AH. Guidance for industry and Food and Drug Administration staff: class II special controls guidance document: repetitive transcranial magnetic stimulation (rTMS) systems. Food and Drug Administration; 2011. Available from: https://www.fda.gov/files/medical\%20devices/published/Guidancefor-Industry-and-FDA-Staff---Class-IISpecial-Controls-Guidance-Document-Computerized-Labor-MonitoringSystems-\%28PDF-Version\%29.pdf 